\title{
Challenges in the diagnosis and treatment of extrauterine leiomyomas: case series
}

\author{
Tang Yafang ${ }^{1}$, Ravichandran Nadarajah ${ }^{2 *}$
}

\author{
${ }^{1}$ Department of Obstetrics and Gynaecology, K. K. Women's and Children's Hospital, Singapore \\ ${ }^{2}$ Department of Gynaecological Oncology, Singapore General Hospital, Singapore
}

Received: 15 August 2021

Revised: 07 September 2021

Accepted: 26 November 2021

\section{*Correspondence:}

Dr. Ravichandran Nadarajah,

E-mail: Ravichandran.nadarajah@singhealth.com.sg

Copyright: () the author(s), publisher and licensee Medip Academy. This is an open-access article distributed under the terms of the Creative Commons Attribution Non-Commercial License, which permits unrestricted non-commercial use, distribution, and reproduction in any medium, provided the original work is properly cited.

\begin{abstract}
Extrauterine leiomyoma is a rare disease with multiple potential metastasizing sites. Pathogenesis of this condition remains debatable with previous myomectomy a considerable risk factor. Diagnosis and treatment remain a challenge due to diverse presenting symptoms and the initial involvement of various specialties, which often cause distress in these patients. In this article, we present one case of pulmonary leiomyomas on expectant management, one case of wide-disseminated extrauterine leiomyomas including the bones, spines, soft tissues and peritoneum who underwent decompression laminectomy and biopsy of paravertebral mass and one case of retroperitoneal leiomyoma who underwent resection of the lesion.
\end{abstract}

Keywords: Extrauterine leiomyoma, Metastasis, Diagnosis, Treatment

\section{INTRODUCTION}

Uterine leiomyoma is a benign gynecologicaltumor that results from clonal proliferation of uterine smooth muscle cells. It is the most prevalent benign gynecologicaltumor and affects up to $30 \%$ of women above the age of $30 .{ }^{1}$ However, extrauterine leiomyomas are a rare entity with complex pathogenesis and the diagnosis and management of which can be challenging. Pulmonary leiomyomas are the most common. ${ }^{2}$ Other sites include bone, spine, lymph nodes, retroperitoneum, intravascular space and heart. ${ }^{3}$ These benign metastasizing leiomyomas occur more commonly in premenopausal women with previous myomectomies but they were also found in women with unresected uterine leiomyomas and postmenopausal women. Diagnosis of this condition can be extremely challenging especially when the site of disease is very unusual or with difficult access. Due to the diverse presenting symptoms, it is common that the primary doctors taking care of these patients are not familiar with this condition which may result in extensive investigations, delay in diagnosis and patient anxiety and distress. Therapeutic options for patients are heterogenous. Management primarily consisted of diagnostic resection with subsequent observation, complete surgical resection and hormonal treatment. ${ }^{4}$ In this article, we present 3 cases of extrauterine leiomyomas at different sites. All patients went through eventful emotional journeys while sinister differentials being discussed and eventually excluded. Treatment was individualized based on the site and extent of disease in each case, after multidisciplinary discussions.

\section{CASE SERIES}

\section{Case 1}

A 48-year-old Chinese female was referred to emergency department in August 2017 for incidental CXR findings of multiple round opacities in both lung fields. Patient 
had no respiratory symptoms. She had regular menses and no complaints of abdominal, urinary or bowel symptoms. She had previous open myomectomy in 2006. Her family history was significant for brain tumor in her father. Physical examination was unremarkable. She was admitted under internal medicine for further investigation. Repeated CXR showed randomly distributed myriad nodules in both lungs highly redolent of metastatic deposits from an occult neoplasm. CT chest, abdomen and pelvis showed multiple randomly scattered pulmonary nodules of varying sizes suggesting bilateral lung metastases with no apparent primary tumor (Figure 1A). Impression of possible metastatic disease was conveyed to patient and CT guided biopsy was proposed for a definitive diagnosis under respiratory medicine. Biopsy revealed spindle cell lesions with smooth muscle derivation and favor pulmonary benign metastasizing leiomyoma. US pelvis showed adenomyotic uterus with $3.3 \mathrm{~cm}$ adenomyoma, a few sub-centimeter fibroids and normal ovaries. Patient was then reviewed by gynecological oncologist. Options of surveillance, hormonal treatment and surgery were discussed. Decision was made for surveillance with yearly CT thorax as she was asymptomatic at peri-menopausal age. Patient remained asymptomatic and subsequent yearly CT chest showed largely stable nodules with some fluctuation. Last CT scan in 2021 showed slightly increased size in a few nodules (Figure 1B). As patient was asymptomatic and having perimenopausal pattern of menses, decision was made to continue expectant management.

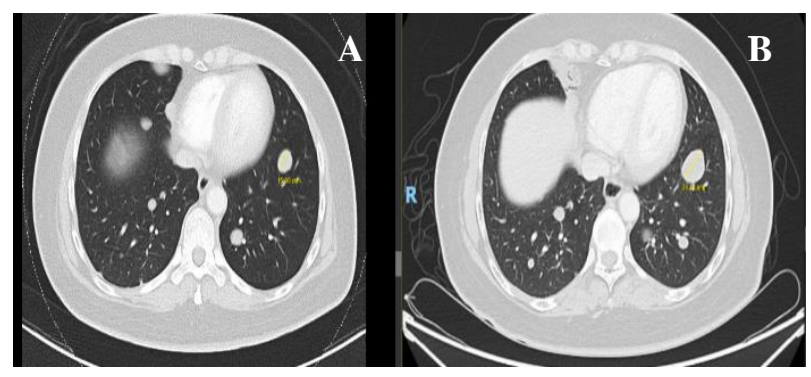

Figure 1: Radiographic images of the chest, A) CT chest showing multiple randomly scattered large pulmonary nodules at diagnosis in 2017, B) CT chest at last follow up visit in May 2021, showing interval increase in the lung nodules.

\section{Case 2}

A 46-year-old premenopausal female presented to the emergency department in January 2021 for 3-week history of back pain at the left paraspinal area. She had loss of appetite for a month but no other constitutional symptoms. Past medical and surgical history included benign breast lump and laparoscopic myomectomy in 2013 for a $9 \mathrm{~cm}$ fibroid which was complicated by recurrence in 2017. Family history was non-contributory except for cervical cancer in her mother. Physical examination showed multiple firm lesions on bilateral arms and back, 20-week-size bulky uterus and paraspinal tenderness at T12 region. She had previously visited a primary care physician who performed MRI of the spine which showed suspicious osseous lesions in the spine, sacrum and ribs, as well as enhancing lesions in the abdominal and pelvic cavity, muscles and subcutaneous tissues, suspicious for metastases. A pathological fracture at T12 causing severe spinal canal narrowing was also evident. She was initially admitted under orthopaedic surgery. CT of the chest, abdomen and pelvis was performed and impression was possible metastatic gynecological malignancy based on findings of a large abdominopelvic mass $(23 \times 14.6 \times 11 \mathrm{~cm})$ (Figure 2A) and extensive lesions in the peritoneum, subcutaneous tissues, muscles, bones, aortocaval region (Figure 2C) and small nodules in the lungs. Leiomyosarcoma was one consideration. Due to the compression fracture of T12 and spinal stenosis, patient was counselled for immediate surgery.

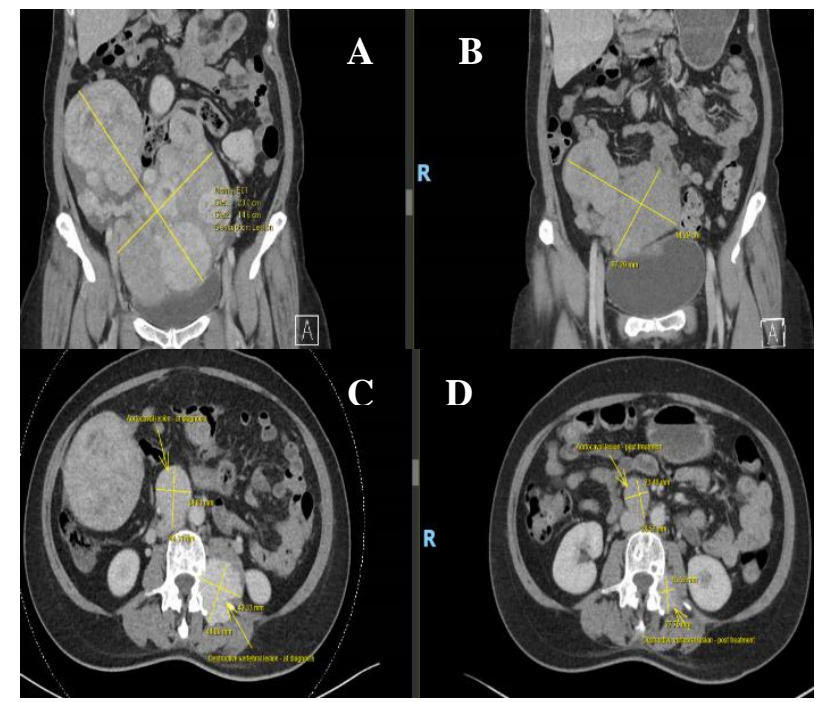

Figure 2: Radiographic images of the chest, abdomen and pelvis, A) large abdominopelvic mass at diagnosis, B) smaller abdominopelvic mass 3 months after hormonal treatment, $C$ ) representative aortocaval and vertebral lesions at diagnosis, D) smaller aortocaval and vertebral lesions 3 months after hormonal treatment.

She underwent posterior decompression laminectomy of T11/T12 with instrumentation and stabilization of T10T12. Excision of multiple vertebral/paravertebral lesions (largest $5 \times 3 \mathrm{~cm}$ ) was performed. Histology revealed leiomyoma with no significant cytologic atypia. ER and PR staining was positive. The case was referred to surgical oncology and discussed at sarcoma tumor board. The panel concluded that leiomyosarcoma cannot be excluded although histology showed minimal atypia and low mitotic activity and recommended consideration of radiation therapy to spine, debulking of the abdominal mass followed by hormonal treatment. As the possible origin of tumor was gynecological, patient was also referred to gynecological oncology. The gynecological oncology tumor board agreed that the nature of the tumor 
was leiomyoma with no atypia or any features to suggest leiomyosarcoma and therefore recommended ovarian suppression without radiation therapy. After thorough discussion with patient, she was started on gonadotropin releasing hormone $(\mathrm{GnRH})$ agonist as well as letrozole. Follow up CT scan in Apr 2021 showed reduced sizes of the uterus and the metastatic deposits (Figure 2B, D). Decision was made to continue hormonal therapy.

\section{Case 3}

A 49-year-old female was on regular follow up for fibroids in a restructured hospital. She had previous laparoscopic myomectomy with power morcellation in 2005. Subsequently, she has been on follow up for stable fibroids. She had regular menses and was otherwise asymptomatic. She has significant history of appendectomy and cholecystectomy. During her routine surveillance ultrasound in Aug 2019, a $4.4 \mathrm{~cm}$ solid mass was noted in close relation to cervix and rectum suspicious for rectal adenocarcinoma. MRI showed a presacral predominantly solid mass separate from the adjacent cervix and rectum with a definite fat cleft seen between these structures (Figure 3).

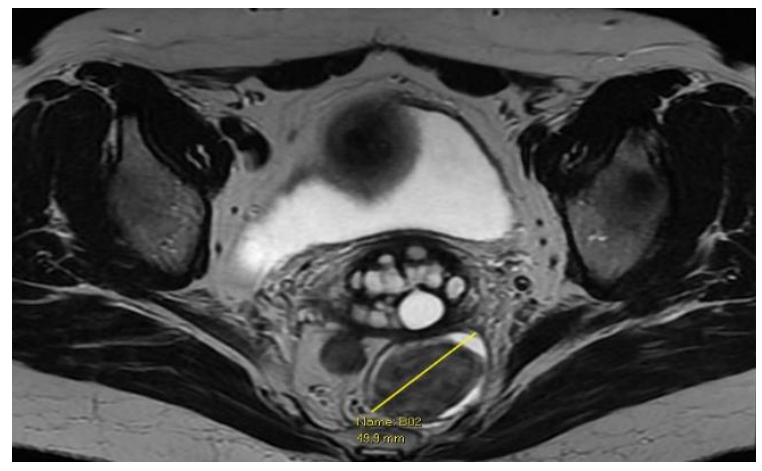

Figure 3: Radiographic images of the pelvis. T1weighted images of the coronal view of MRI pelvis, showing a pre-sacral predominantly solid mass separate from the adjacent cervix and rectum with a definite fat cleft seen between these structures.

Differentials include neurogenic tumor, extra-gonadal germ-cell and sex-cord stromal tumors. Patient was then seen by colorectal surgeon and per-rectal exam revealed a $5 \mathrm{~cm}$ well circumscribed left pararectal mass, extrinsic to rectum. Colonoscopy showed normal findings. In view of uncertain nature and unusual location of the mass, bulky uterus with multiple fibroids interfering the access to the mass, patient was counselled for hysterectomy with bilateral salpingectomy (with conservation of ovaries) and resection of the pararectal mass. She was keen and underwent surgery in December 2019. Uterus and retroperitoneal myoma were delivered intact vaginally. Histology showed benign leiomyoma. Patient underwent uneventful post-operative recovery and remained well since then.

\section{DISCUSSION}

Extrauterine leiomyomas occur more commonly in women of perimenopausal age with previous surgical resection of uterine leiomyoma. A review article reported average age of primary leiomyoma surgery at 38.5 years and mean age of diagnosis of metastasizing leiomyoma at 47.3 years. $^{5}$ Other studies speculated the estimated time of occurrence from initial surgery to time of extrauterine leiomyoma diagnosis to be $10-15$ years. ${ }^{6}$ Although uncommon, extrauterine leiomyomas were also found in patients with no previous uterine myoma surgeries. ${ }^{7}$ Sites of extrauterine deposits are diverse, including lungs, bone, spine, retroperitoneum, intravascular, heart and so on. The pathogenesis of this disease remains controversial but there are a few proposed mechanisms. Coelomic metaplasia; It is likely that these tumors originate from subcoelomic mesenchymal cells which underwent metaplasia and differentiated into myofibroblasts under hormonal influence. ${ }^{8}$ Hematological and lymphatic transport of myomatous tissue cells; as most patients with extrauterine leiomyoma have previous history of myomectomies, tumor could potentially gain venous access from surgical trauma and cause hematogenous spread to other sites. ${ }^{9}$ Fragments of leiomyoma could also detach during these procedures, enter the lymphatic channel and seed pelvic and paraaortic lymph nodes. Peritoneal seeding; animal studies showed that fibroid tissues were able to implant and grow after being inserted into the subcutaneous tissue. ${ }^{8}$ Peritoneal leiomyomatosis may evolve by parasitic implantation or infiltration from adherent subserous leiomyomas to the surrounding structures. ${ }^{12}$ Iatrogenic seeding from power morcellation may also play an important part. One study reviewed 600 laparoscopic gynecologic surgeries and found that peritoneal myomas occur in $1.2 \%$ of myomectomies using power morcellation. $^{13}$ As presentation of extrauterine leiomyoma can be delayed a decade after surgery, more cases are expected in the coming years despite the recent introduction of contained morcellation. Finally, metastasizing leiomyosarcoma should always be excluded. Diagnosis of such condition can be challenging as it is a rare entity and there are more common differentials. Case 2 is rather rare as the myomatous deposits were extremely extensive, involve multiple organs and cause severe symptoms. Patient was cared by multiple specialties and various investigations were carried out before a diagnosis was made. Definitive diagnosis always relies on histopathologic examination, as in all our presented cases. In some cases, biopsy may not be easily performed due to difficult access and biopsy result may not be conclusive due to limited sampling. Surgical excision might be required in these cases such as in case 3. Awareness of this condition across specialties and high index of suspicion are crucial during investigation to avoid unnecessary tests and patient anxiety. Management of extrauterine leiomyomas varies depending on the site of lesions. Solitary pulmonary lesions can be treated with curative surgical excision 
while multiple pulmonary leiomyomas are usually treated with hormonal therapy or active surveillance after obtaining histological diagnosis. ${ }^{13}$ Disease control or regression with hormonal manipulation were reported in $79 \%$ of patients in literature. ${ }^{13} \mathrm{GnRH}$ agonists has been reported to either maintain the lung nodule sizes or lead to shrinkage of the nodules. ${ }^{14}$ Progesterone has been shown effective in both prophylaxis against recurrence and regression of extrauterine leiomyomas. ${ }^{15}$ Estrogen receptor antagonist such as tamoxifen and aromatase inhibitors have also been explored and there were reports showing effect to maintain stable nodules or even regression. ${ }^{15}$ However, postmenopausal women may be less amenable to hormonal treatment. Two out of three postmenopausal women had disease progression after hormonal treatment in a case series. $1^{2}$ Active surveillance with regular CT scan is recommended to detect disease progression and complications such as parenchymal loss. Pelvic and retroperitoneal nodules are more commonly treated surgically especially when it is solitary or patient is symptomatic. In asymptomatic patient with a clear histological diagnosis from biopsy, expectant management is a reasonable option. Hormonal treatment as mentioned earlier can be considered as well. ${ }^{4}$ However, to date there is still no consensus or standardized treatment for the management of extrauterine leiomyomas.

\section{CONCLUSION}

Extrauterine leiomyomas are a rare entity with complex pathogenesis. Diagnosis remains a challenge due to unusual locations of lesions and invasive biopsies or excisions are usually required. The psychological impact on patient who are initially misdiagnosed as malignancy should not be underestimated. Meanwhile, malignancy is always a crucial differential to consider and exclude in these cases. It is important to raise awareness of this condition across different specialties and ensure the diversity of metastasizing sites and extent of disease are recognized by them to facilitate timely diagnosis and reduce patient anxiety. Accurate diagnosis enables the clinician to offer proper counselling on treatment options. The best treatment option should be discussed between the patient and physician based on individual clinical scenario and considering the benefits and risks of each treatment option. Further research is still required to develop more standardized treatment protocols.

\section{ACKNOWLEDGEMENTS}

We would like to thank department of radiology, Singapore general hospital for providing the radiographic images.

Funding: No funding sources

Conflict of interest: None declared

Ethical approval: Not required

\section{REFERENCES}

1. Khan AT, Shehmar M, Gupta JK. Uterine fibroids: current perspectives. Int J Womens Health. 2014; 6:95-114

2. Raś R, Książek M, Barnaś E. Benign metastasizing leiomyoma in triple location: lungs, parametria and appendix. Prz Menopauzalny. 2016; 15:117-21.

3. Kim YN, Eoh KJ, Lee JY. Aberrant uterine leiomyomas with extrauterine manifestation: intravenous leiomyomatosis and benign metastasizing leiomyomas. Obstet Gynecol Sci. 2018;61:509-519.

4. Nasu K, Tsuno A, Takai N, Narahara H. A case of benign metastasizing leiomyoma treated by surgical castration followed by an aromatase inhibitor, anastrozole. Arch Gynecol Obstet. 2009;279:255-7.

5. Barnaś E, Książek M, Raś R, Skręt A, SkrętMagierøo J, Dmoch-Gajzlerska E. Benign metastasizing leiomyoma: A review of current literature in respect to the time and type of previous gynecological surgery. PLOS ONE. 2017;12: e0175875.

6. Wei WT, Chen PC. Benign metastasizing leiomyoma of the lung: A case report and literature review. Oncol Lett. 2015;10:307-12.

7. Usman $\mathrm{Y}$, Iftikhar $\mathrm{O}$, Ishaq $\mathrm{MK}$, Awab A. Pulmonary benign metastasizing leiomyoma, why metastasizes if benign. Chest. 2015;148:627A.

8. Hoynck van Papendrecht HP, Gratama S. Leiomyomatosis peritoneal is disseminata. Eur $\mathrm{J}$ Obstet Gynecol Reprod Biol. 1983;14:251-259.

9. Wentling GK, Sevin BU, Geiger XJ, Bridges MD. Benign metastasizing leiomyoma responsive to megestrol: case report and review of the literature. Int J Gynecol Cancer. 2005;15:1213-7.

10. Hassan MH, Eyzaguirre E, Arafa HM, Hamada FMA, Salama SA, Al-Hendy A. Memy I. a novel murine model for uterine leiomyoma using adenovirus enhanced human fibroid explants in severe combined immune deficiency mice. Am J Obstet Gynecol. 2008;199:156.e1-8.

11. Rader JS, Binette SP, Brandt TD, Sreekanth S, Chhablani A. Ileal hemorrhage caused by a parasitic uterine leiomyoma. Obstet Gyneco.1 1990;76:531-4.

12. Cucinella G, Granese R, Calagna G, Somigliana E, Perino A. Parasitic myomas after laparoscopic surgery: an emerging complication in the use of morcellator? Description of four cases. Fertil Steril. 2011;96:e90-6.

13. Miller J, Shoni M, Siegert C, Lebenthal A, Godleski $\mathrm{J}$, McNamee C. Benign Metastasizing Leiomyomas to the Lungs: An institutional case series and a review of the recent literature. Ann Thorac Surg. 2016;101:253-8.

14. Mogi A, Hirato J, Kosaka T, Yamaki E, Kuwano H. Benign metastasizing leiomyoma of the lung: report of a case. Gen Thorac Cardiovasc Surg. 2013;61: 719-22. 
15. Bakkensen JB, Samore W, Bortoletto P, Morton CC, Anchan R. Pelvic and pulmonary benign metastasizing leiomyoma: A case report. Case Rep Women Health. 2018;18:e0061.
Cite this article as: Yafang T, Nadarajah R. Challenges in the diagnosis and treatment of extrauterine leiomyomas: case series. Int J Reprod Contracept Obstet Gynecol 2022;11:232-6. 